Published in final edited form as:

Bioessays. 2007 July ; 29(7): 668-677. doi:10.1002/bies.20601.

\title{
Evolution of the cytoskeleton
}

\author{
Harold P. Erickson \\ Department of Cell Biology, Duke University Medical Center, Durham, NC 27710-3709. E-mail: \\ h.erickson@cellbio.duke.edu
}

\section{Summary}

The eukaryotic cytoskeleton appears to have evolved from ancestral precursors related to prokaryotic FtsZ and MreB. FtsZ and MreB show 40-50\% sequence identity across different bacterial and archaeal species. Here I suggest that this represents the limit of divergence that is consistent with maintaining their functions for cytokinesis and cell shape. Previous analyses have noted that tubulin and actin are highly conserved across eukaryotic species, but so divergent from their prokaryotic relatives as to be hardly recognizable from sequence comparisons. One suggestion for this extreme divergence of tubulin and actin is that it occurred as they evolved very different functions from FtsZ and MreB. I will present new arguments favoring this suggestion, and speculate on pathways.

Moreover, the extreme conservation of tubulin and actin across eukaryotic species is not due to an intrinsic lack of variability, but is attributed to their acquisition of elaborate mechanisms for assembly dynamics and their interactions with multiple motor and binding proteins. A new structure-based sequence alignment identifies amino acids that are conserved from FtsZ to tubulins. The highly conserved amino acids are not those forming the subunit core or protofilament interface, but those involved in binding and hydrolysis of GTP.

\section{Historical introduction}

\section{Discoveries of the prokaryotic cytoskeleton}

Before 1990, the cytoskeleton was thought to have evolved only in eukaryotes. Relatives or homologs of actin, tubulin or intermediate filaments were unknown in bacteria or archaea. There were sporadic reports of candidates for bacterial actin and tubulin in the 1970s and 1980s but, apart from some intriguing images of microtubules in certain bacteria,(1) these turned out to be wrong and/or were not followed up.

The first suggestions of a bacterial homolog of tubulin appeared in 1992. Three groups independently discovered that the bacterial cell division protein FtsZ bound and hydrolyzed GTP, as does tubulin, and had a seven-amino-acid sequence, GGGTGTG, virtually identical to the "tubulin signature sequence".(2-4) Mukherjee and Lutkenhaus(37) then extended the sequence alignment to find a dozen additional amino acids that were completely conserved in FtsZ and in $\alpha, \beta$ and $\gamma$ tubulins. They also showed that FtsZ assembled in vitro into filamentous structures. Erickson et al.(5) found that FtsZ assembled into protofilaments that could adopt two conformations, straight and curved, similar to the straight protofilaments of the microtubule wall and the tubulin rings that peel away from microtubules during disassembly. Any question of homology was resolved when tubulin and FtsZ were found to have virtually identical structures at the level of protein folding. $(6,7)$

If one looks for bacterial relatives of tubulin or actin using a simple computer search for sequence similarity, nothing will be found. In 1992 Bork et al.(8) used a sophisticated structurebased sequence alignment, and they discovered that bacteria did have genes related to actin. Actin is a member of a larger superfamily that includes sugar kinases, the chaperone hsp70, and the actin subfamily. These three subfamilies show no recognizable sequence identity to 
each other in normal pairwise alignments, but when the structures of hexokinase, hsp70 and actin were determined by X-ray crystallography, they were found to have virtually identical folding patterns. Structural biologists interpret this to mean that the three subfamilies evolved from a common protein ancestor.(9) Sugar kinases and hsp70 were already known to exist in both bacteria and eukaryotes because of their clear sequence similarity: the bacterial proteins are 50-60\% identical to their eukaryotic homologs.(10) But the closest bacterial proteins to eukaryotic actin showed less than $15 \%$ identity,(8) which is too low for reliable identification. However, the more-sophisticated alignment of Bork et al.(8) found three bacterial proteins that appeared to be relatives of actin-FtsA, MreB and StbA (now called ParM).

Remarkably, this pioneering study was not followed up for almost ten years. FtsA, a cell division protein known to co-localize with FtsZ in the cytokinetic ring, was the first to be explored. The group of Löwe determined the X-ray crystal structure of FtsA, which confirmed that it was in the actin superfamily(11) and, probably, most closely related to the actin subfamily, rather than sugar kinases or hsp70. The strongest evidence for its relation to actin is its ability to assemble into filaments. FtsA from Escherichia coli can form homodimers (12) and, in some circumstances, more extended filaments.(13) FtsA from Streptococcus pneumoniae can assemble in vitro into long spiral filaments.(14)

In 2001 two laboratories provided definitive evidence that bacterial MreB assembles into cytoskeletal filaments that are clear analogs of eukaryotic actin. Jones, Carballido-López and Errington(15) demonstrated the presence of helical filaments in B. subtilis at the level of light microscopy. van den Ent, Amos and Löwe(16) used electron microscopy and X-ray crystallography to demonstrate assembly of MreB into actin-like filaments.

The third filament of the eukaryotic cytoskeleton, intermediate filaments, has at least one homolog in bacteria. The protein crescentin, whose sequence and domain structure are unmistakably those of an intermediate filament protein, is responsible for the crescent shape of Caulobacter crescentus.(17) In this case, however, the protein has been found only in this single species, and it seems likely that $C$. crescentus acquired it in a recent horizontal gene transfer from a eukaryote.

\section{Evolution of eukaryotes}

It is now widely agreed that bacteria, archaea and eukarya are three separate domains of life, but there is still much dispute over how they are related to each other in early evolution. It is clear that eukaryotes have many genes and important pathways closely related to archaea, (18) but it is still debated how and when eukaryotes separated from bacteria and archaea. One theory, which was originally proposed many years ago(19) and is seeing a rebirth from recent genomics analysis, $(20,21)$ is that bacteria, archaea and eukarya separated very early from a common ancestor. The three lines evolved independently and, later in evolution, the eukaryotic line acquired mitochondria. The new evidence for the early emergence of a eukaryotic line is the identification of a group of proteins with no homologs in bacteria and archaea.(20,21) This hypothesis is, however, still controversial. Alternatively, a number of groups have proposed that early evolution involved only two lines, bacteria and archaea, and that the eukaryotic line began with the acquisition of a bacterial endosymbiont by an archaeon.(22) There are a number of variations of this theory.(23) A third proposal is that early evolution involved only one line, bacteria, and that archaea and eukarya separated from the bacterial line relatively recently. (24) These and other theories are discussed in the recent review of Embley and Martin.(23) In addition, I recommend Kurland et al.,(21) which is not included in the above review, and Cavalier-Smith(24) for lively presentation of their rather divergent views of the evolution of eukaryotes. 
Mitochondria are widely recognized as bacterial endosymbionts but, until recently, their capture was thought to be a late event in the evolution of eukaryotes. This idea was based on studies of several eukaryotes that appeared to have no mitochondria, and thus were thought to have come from a primitive eukaryote line before the acquisition of mitochondria. However, all of these organisms are now known to have hydrogenosomes or mitosomes, organelles derived from mitochondria. There are currently no known eukaryotes that lack mitochondria or organelles derived from mitochondria. This has renewed the idea that the acquisition of the mitochondrion may have been a key and perhaps very early step in the evolution of eukaryotes. This new evidence and analysis are clearly argued by Embley and Martin.(23)

In 1970 Roger Stanier(25) proposed "that the progressive structural evolution of the eukaryotic cell received its initial impetus from the acquisition of a novel cellular property, the capacity to perform endocytosis". He noted that this would provide "a new biological means for obtaining nutrients: predation on other cells". This would be especially important for the eukaryotic line, which relies (apart from mitochondria) on the inefficient glycolysis pathway for energy.(25) Phagocytosis would eventually provide the means for capturing the bacteria that became mitochondria and chloroplasts, which provided much more efficient energy supplies. The concept that acquisition of phagocytosis was the key step in the early evolution of eukaryotes is now a fundamental part of almost every modern treatment of evolution.(20, $21,24,26,27)$

\section{The enigma of the divergence of the bacterial and eukaryotic cytoskeletons}

R.F. Doolittle(28) pointed out an enigma about tubulin and FtsZ - tubulins are among the most slowly diverging proteins in eukaryotes, yet they are so divergent from their bacterial homolog FtsZ as to be practically unrecognizable. What is the reason for this extreme divergence from its precursors, and the virtual stasis across all eukaryotic species? D.M. Faguy and W.F. Doolittle(29) (a different Doolittle) addressed this conundrum from a different perspective. Bacterial FtsZs are $40-50 \%$ identical in sequence even across very divergent species. Archaeal FtsZs show a similar level of identity to each other and to bacterial FtsZs. (See Vaughan et al. (30) for an extensive analysis of the evolution of FtsZs and comments on the few exceptions to this conservation.) In contrast, $\beta$ tubulins show $75-85 \%$ sequence identity across most animals, plants and fungi (with a few exceptions $(31,32)$ ). Why are tubulins so much more conserved than FtsZ across modern eukaryotic species, and so extremely divergent from their ancestral FtsZ?

A similar situation exists for MreB and actin. Bacterial MreBs are generally about $40 \%$ identical in sequence even across diverse species, similar to FtsZ. Eukaryotic actins are even closer to each other in sequence than tubulins; actin from rabbit and yeast are 88\% identical. Yet MreB and actin show less than $15 \%$ sequence identity to each other. R.F. Doolittle and A. York(10) highlighted the enigma by noting that hsp70 and sugar kinases, the other branches of the actin superfamily, show $\sim 50 \%$ sequence identity between eukaryotic and bacterial homologs. If hsp70 is so highly conserved from bacteria to eukaryotes, why have actin and MreB diverged so far apart in sequence?

Both groups pointed out possible solutions to the enigma. Among them was that tubulin and actin have evolved into structures that perform very different functions from their precursors. hsp70 and sugar kinases are conserved between prokaryotes and eukaryotes because they have retained the same functions in both. In this essay, I will elaborate on this idea and speculate on the pathway for the evolution of tubulin and actin from FtsZ and MreB. 


\section{Evolution of FtsZ and tubulins}

Davis(33) has suggested that FtsZ is a very ancient protein. He notes that arginine, lysine, phenylalanine, tyrosine and histidine are rare in FtsZ sequences, and tryptophan is almost nonexistent. Davis argues that these amino acids were the last to be added to the genetic code, and suggests that FtsZ evolved as a functional protein before the genetic code was complete. This makes sense, because a mechanism for cell division would be needed in the earliest stage of development of a cellular life form. A division mechanism based on FtsZ was apparently quite successful, because FtsZ has been retained by almost every modern species of bacteria and archaea. FtsZ is missing from the bacterial species Ureaplasma urealyticum, the phylum Chlamydiae and Planctomycetes and the archaeal division Crenarchaeota. If FtsZ is truly an ancient protein, it is likely that these species originally had it, but lost it after developing an alternative (and still unknown) mechanism for division.

It seems clear that FtsZ originated in the common ancestor (or whatever was the earliest cellular life form) and was passed to bacteria and euryarchaea, where it is used in almost all modern species. This makes it likely that FtsZ was also used for division in the earliest eukaryotic line, whatever its origin. Eukaryotes later evolved an actin-based machine for cytokinesis, and eukaryotic FtsZ underwent a drastic change as it evolved into tubulin. This will be discussed below, after a consideration of what is conserved between FtsZ and tubulins.

\section{Amino acids highly conserved from FtsZ to tubulin are those involved in GTP binding and hydrolysis}

In order to further study the evolution of tubulins from FtsZ, I have prepared a new sequence alignment, relying on recent structure-based pairwise alignments,(34-36) and have identified amino acids that are highly conserved in FtsZ and in $\alpha, \beta$ and $\gamma$ tubulins. This was originally done by Mukherjee and Lutkenhaus,(37) and the resulting sequence identities, although limited, provided the strongest early argument for homology of FtsZ and tubulin. While that alignment remains largely correct in identifying highly conserved amino acids, it is less accurate in non-conserved regions. At the time of this early attempt, there was no structural information for any of the proteins, but we now have atomic structures for $\alpha, \beta$ and $\gamma$ tubulins, and several FtsZs. The new sequence alignment is shown in Fig. 1, and the conserved amino acids are listed in Table 1. (A DNAstar or GCG pileup file of the alignment will be made available upon request).

Fig. 2 shows the location of these conserved amino acids on the structure, and makes an important and novel point. The conserved amino acids are almost all clustered on the top or bottom surfaces. These surfaces form the interfaces that make the protofilament, which is common to FtsZ and tubulin.(38-40) I had initially concluded that these conserved amino acids were those making the interface contacts. However, this is not the case. Nogales et al(41) list more than 40 amino acids that make contacts across the interface of $\alpha$ and $\beta$ tubulin (Table 2 of their paper), and only four of them are among the conserved amino acids in Table 1. More importantly, each of those four amino acids contacts the GTP molecule. This correlation can be extended as the primary basis for conservation. Almost all of the conserved amino acids (Table 1) directly contact the GTP molecule, either forming the GTP-binding pocket on the top, or the GTP-contacting synergy loop $(34,42)$ on the bottom. Apart from D158 and two hydrophobic core amino acids (I13 and F99), the remaining conserved amino acids are close to the GTP and appear to be supporting the structure of the binding pocket or the synergy loop.

D212 is completely conserved in FtsZ and is essential for GTP hydrolysis. $(43,44)$ In $\alpha$ tubulin, which catalyzes the GTP of $\beta$ tubulin below, there is a conservative change to E254, and this 
glutamate is also essential for catalysis. In $\beta$ tubulin, which does not catalyze hydrolysis, this residue is $\mathrm{K}$ and, in $\gamma$ tubulin, which has low or negligible GTP hydrolysis, $(35,45)$ it is G or S.

D158 is a surface residue that is not important for protein folding and is not close to the protofilament interface. Its conservation in all FtsZs and tubulins is a mystery. Mutating it to alanine produced no obvious phenotype in $E$. coli FtsZ.(46) In yeast $\beta$ tubulin, the double mutant E194A/D197A caused benomyl resistance but cells were viable.(47) In yeast $\alpha$ tubulin, a triple mutant containing these two plus H198A was recessive lethal, but it is not known which mutation caused the defect.(48)

This conclusion that the amino acids involved in binding and hydrolysis of GTP are highly conserved is a new one for FtsZ and tubulin. However, the same conclusion has recently been drawn for MreB and actin. Amino acids involved in binding the ATP molecule are highly conserved, while those involved in filament contacts are not.(16) This leads to a somewhat remarkable generalization. Although previous studies have found that surface amino acids involved in protein-protein interfaces are more highly conserved than other surface amino acids,(49-51) it appears that over long periods of evolution, interfaces can be mutated at almost all contacting amino acids. Apparently a mutation in the interface can be compensated by a second mutation nearby. Something similar occurs for interior amino acids involved in folding the protein subunit, as these are also not conserved between tubulins and FtsZ.

\section{What drives sequence divergence from FtsZ to tubulins?}

In the alignment in Fig. 1, the sequence identity between FtsZs and tubulins is less than 10\%. FtsZ itself is much more highly conserved across even very diverse bacterial species. The conserved core of $E$. coli $\mathrm{FtsZ}$ (which ignores the C-terminal tail beyond amino acid 312 ) shows $53 \%$ and $46 \%$ sequence identity to FtsZ from Bacillus subtilis and Mycoplasma pulmonis, and $46 \%$ identity to FtsZ1 of the archeon Halobacterium. These species are extremely divergent from each other, so how do we explain the apparently high level of sequence conservation? I suggest that, after FtsZ arose in the common ancestor of bacteria and archaea, it evolved a mechanism that was quite efficient for cytokinesis. As FtsZ later evolved in the diverging species, it preserved this mechanism largely unchanged. (In this regard, it is noteworthy that FtsZ from B. subtilis and M. pulmonis can function for division in E. coli(52)). Apparently this core $45-55 \%$ sequence identity is sufficient to preserve the features needed for the cell division mechanism.

I conclude further, as previously suggested, $(10,28)$ that the extreme divergence of tubulins and FtsZ arose when a redundant FtsZ in early eukaryotes lost the constraints needed to do cell division and began evolving a completely different function, to form microtubules. The redundant FtsZ could have come from a gene duplication, or after actin replaced FtsZ for cytokinesis.

While freed from its full role in cell division, the newly evolving FtsZ would preserve its ability to assemble into protofilaments. These protofilaments would have some rigidity, and this mechanical property might have been adopted to some role outside of the $\mathrm{Z}$ ring. Mechanical strength would be enhanced by lateral contacts that further assembled them into bundles or small sheets and, if these sheets adopted a curvature, their mechanical strength would be further increased. Eventually, closure into a cylindrical wall of 13 protofilaments would complete the design of the optimal rigid cytoskeletal filament, the microtubule.

The acquisition of a completely new function, assembling a microtubule rather than carrying out cytokinesis, can explain the extreme sequence divergence between tubulin and its FtsZ ancestor. What can explain the extremely high level of conservation of $\alpha$ and $\beta$ tubulins across diverse eukaryotes? This may be partly due to constraints in assembling the cylindrical 
microtubule, but it is probably due more to additional evolutionary constraints. The microtubule has mechanisms for nucleated assembly from the centrosome, and complex mechanisms producing dynamic instability. These must impose additional constraints. Finally, microtubules did not remain as passive mechanical struts, but became the tracks for motor molecules, kinesins and dyneins. The evolution of these motors is itself a fascinating story, $(53,54)$ but my focus here is on the cytoskeleton. As motors developed the ability to bind and move along the microtubule surface, this must have involved co-evolution of the microtubule surface to facilitate these interactions. The evolution of motors and co-evolution of $\alpha$ and $\beta$ tubulin must have occurred early in the eukaryotic lineage, since all eukaryotes have kinesins and dyneins. An important consideration is that most species have multiple different kinesins and dyneins, as well as non-motor microtubule-binding proteins.

If the motors and binding proteins were the major constraining factor, one might expect that the outside surface of the microtubule would be the most highly conserved. To explore this, I identified 31 weakly conserved amino acids, as ones that were different in pig, yeast and arabidopsis alpha tubulins. Two of these non-conserved amino acids were on the front surface, three were on the back (the inside of the microtubule, with no known binding partners), nine were on the lateral surfaces, and the rest were buried. The conclusion is that the conservation extends over the entire molecule, not just the front surface. The overall mechanical properties, the complex mechanisms needed for dynamic assembly, and binding of motors have apparently been optimized over all surfaces and the core of the tubulin subunits.

\section{Lessons from bacterial tubulins and the extended tubulin superfamily}

One might wonder whether the high level of sequence conservation of $\alpha$ and $\beta$ tubulin could be intrinsic to the tubulin structure itself. Two observations suggest that it is not. First, Jenkins et al.(55) discovered two genes in Prosthecobacter encoding proteins obviously related to $\alpha$ and $\beta$ tubulins. Several arguments favor the hypothesis that these bacterial tubulins were acquired by horizontal gene transfer from a eukaryotic host.(56,57) Following this transfer, the tubulins would suddenly be removed from the presence of any of the eukaryotic motors and microtubule-binding proteins, and would be free to rapidly diverge in the bacterial host. Indeed, the bacterial tubulins have lost the ability to make cylindrical microtubules, instead forming pairs and bundles of protofilaments.(56,57) It is still not known how these tubulins function in Prosthecobacter, but their continued expression implies that they are of some benefit to the host.

BtubA and BtubB show 34 and 36\% sequence identity to pig $\alpha$ and $\beta$ tubulin, and 26 and $31 \%$ identity to $\gamma$ tubulin, suggesting a closer relationship to $\alpha$ and $\beta$ tubulins. They show only $9 \%$ identity to FtsZ, similar to other tubulins. The $\sim 35 \%$ sequence identity shows how much tubulin can change when freed from its complex functions and accompanying interactions in eukaryotes.

The second evidence of tubulin's intrinsic ability to evolve is the existence of an extended superfamily of tubulins. In addition to $\alpha, \beta$ and $\gamma$ tubulins, $\delta, \varepsilon, \zeta$ and $\eta$ tubulins(58) have now been identified. These tubulins show only $22-30 \%$ identity to $\alpha$ tubulin.(35) $\gamma$ tubulin is involved in nucleation of microtubules (assembled from $\alpha \beta$ tubulin), and the other tubulins are involved in the structure of centrioles and cilia.(58) This $22-30 \%$ identity of the superfamily members shows that the early tubulin ancestor in the eukaryote line underwent gene duplication, and the redundant genes diverged extensively. Divergence became highly restricted again when each family member acquired its specialized functions.

Finally, we should note the recently discovered genes on large plasmids of Bacillus species that show limited but convincing identity to FtsZ and tubulin. $(59,60)$ These appear to function 
in plasmid partitioning by forming a cytoplasmic filament bundle that binds a plasmid at each end and pushes them to opposite poles. Additional distant relatives of FtsZ in archaea are discussed by Vaughan et al.(30)

\section{Evolution of MreB and actin}

Whereas FtsZ appears to be an ancient protein with no close relatives, MreB belongs to a superfamily with sugar kinases and chaperones as relatives. It seems likely that sugar kinases developed first. These metabolic enzymes would be important for life, whereas MreB functions to determine the rod shape, a function that is useful but not essential. If one of the sugar kinases evolved the ability to assemble into filaments this could lead to MreB.

Certain structural details of MreB are close to actin, and differ from sugar kinases and hsp70. (16) Most importantly, MreB assembles into cytoskeletal filaments with a strong structural resemblance to actin.(16) Since MreB and actin are found widely in bacteria and eukaryotes, respectively, it is reasonable to conclude that this form of cytoskeletal filament originated in the common ancestor of each.

MreB is found only sporadically in archaea. Roeben et al.(61) have argued that these archaeal MreBs likely came from horizontal gene transfers. In this case, MreB may have been lost from the archaeal line early in its development, and re-acquired sporadically by horizontal gene transfer. An archaeal MreB from Thermoplasma has structural characteristics very similar to bacterial MreBs, $(61,62)$ but it constitutes only $0.04 \%$ of the total cell protein. Interestingly, Thermoplasma does have a robust cytoskeleton,(63) but this MreB appears to be too scarce to be its source. The Thermoplasm cytoskeleton may be formed by a novel protein.

In addition to bacterial MreB, distant actin homologs are produced by some bacterial plasmids. $\operatorname{ParM}(64,65)$ and AlfA(66) assemble actin-like filaments (probably forming bi-polar filament bundles) that bind a plasmid at each end and push them to opposite poles, thus achieving efficient segregation of low-copy-number plasmids. ParM and AlfA are as distantly related to each other as they are to actin, FtsA and MreB.(66) It is interesting that some plasmids use actin homologs, and others use tubulin homologs, for this mitosis-like segregation.

\section{Conservation of MreBs and the divergence of actin}

Bacterial MreBs are about 40\% identical in sequence even across diverse species. This level of sequence conservation, which is similar to that of FtsZs, suggests that MreB is performing a similar function in the different species. In all cases examined, one common function is the maintenance of cell shape. In at least some species, MreB also plays a role in chromosome separation, $(67,68)$ but this likely evolved later than the simpler mechanical function. As with FtsZ, I would suggest that the mechanical function of MreB for cell shape was optimized early, and that the $\sim 40 \%$ sequence identity represents the limit of the divergence that is consistent with maintaining this function in different species.

How then did actins diverge so far in sequence from MreB? I think the answer is similar to the case of tubulin, in that an MreB in the eukaryotic line lost its original function and acquired new ones, resulting in extensive evolution. The novel functions would eventually lead to a contractile machine capable of carrying out cell division, various motile functions and phagocytosis. Primitive mechanisms related to these might have been achieved by MreB/actin acting alone as a filament, but the modern actin cytoskeleton involves a multitude of myosin motors and actin-binding proteins.

The family of myosins now numbers at least 37 different varieties.(54) Although much of the elaboration of the myosin family occurred after the divergence of eukaryotic species, three 
myosin subfamilies are found in all eukaryotic divisions, implying a very early separation. (54) Eventually a large number of non-myosin actin-binding proteins arose. "Actin participates in more protein-protein interactions than any known protein."(69) As each of these myosin and actin-binding proteins evolved to optimize its interactions with actin, the actin would coevolve and diverge from its original MreB-derived sequence. These multiple interactions would eventually constrain further divergence. Probably as important as the binding interactions, actin has developed a complex dynamic assembly regulated by ATP hydrolysis, which is in turn regulated by binding proteins.(70) These complex assembly mechanisms, and perhaps related internal protein conformational changes,(71) would all contribute to the constraint on evolution of actin.

As with tubulin, the high level of conservation of actin applies to this one member of the actin family. Eukaryotes have generated a family of ten actin-related proteins, which vary from $17 \%$ to $45 \%$ sequence identity to actin. $(72,73)$ Arp $2 / 3$ functions in actin nucleation and formation of the branched actin network. Most of the other Arps function in the nucleus, in chromatin remodeling. The family of Arps demonstrates that the highly restricted evolution of actin across eukaryotic species is not intrinsic to actin itself, since early in evolution it was able to duplicate and evolve into this very divergent family.

\section{Evolution of phagocytosis}

As noted above, developing a mechanism for phagocytosis has long been postulated as a key step in eukaryotic evolution. $(20,21,24-27)$ Once this machinery was in place, the primitive eukaryote could become a predator with the ability to engulf bacteria and archaea. Much of this engulfment would have been for food, but eventually endosymbiosis would lead to mitochondria and chloroplasts.

Two developments would be important to achieve phagocytosis. First, the organism would have to lose its rigid cell wall, leaving a flexible plasma membrane that could be modulated to project and surround prey. Some archaea and mollicutes have no cell wall and already meet this requirement. The neomuran hypothesis(74) postulates that this might have occurred in bacteria. Second, the organism would need a mechanism for projecting the membrane in a manner that could engulf prey. This would require a cytoskeleton that could produce localized forces.

The eukaryotic actin cytoskeleton can generate force on the membrane by two mechanisms. The first mechanism is a protrusion force generated by the simple act of polymerization.(70) The second involves motor molecules to slide the actin filaments relative to each other or to a membrane. The polymerization-based membrane protrusion mechanism would have developed naturally as a result of actin assembly, while the addition of contractile mechanisms involved many steps to evolve the set of motor molecules and actin-binding proteins.

Could the original phagocytotic mechanism have been based on simple polymerization-based membrane protrusion? Perhaps. Modern mollicutes such as Mycoplasma mobile exhibit a gliding motility on solid substrates, which is based on protrusion of the membrane at the leading edge followed by adhesion to the underlying substrate.(75) One might imagine this gliding cell encountering and sliding over a bit of bacterial debris on the substrate (I suggest debris as an early prey for phagocytosis, because it should be smaller than the gliding cell). If the protrusion force was relaxed or blocked at the site of the debris and maintained around it, a cup could be formed that partially engulfed the debris. If the protrusive forces could be somehow directed, the cup might be closed and the debris engulfed. So I think it is possible that a primitive phagocytosis mechanism could have been achieved by polymerization-based membrane protrusion. 
It should be noted that M. mobile does not have MreB, and the cytoskeletal elements or other mechanisms generating the protrusion forces are unknown. Similarly, the archeon $T$. acidophilum can form a robust cytoskeleton in vitro(63) that is probably not MreB. So we should keep in mind that a primitive phagocytic machine might have been made from a cytoskeletal system unrelated to MreB, and later replaced by the actin system.

Modern eukaryotic cells use the actin-myosin contractile apparatus for several purposes. Phagocytosis and cytokinesis are two that probably evolved early, and either one could have come first. Each of these requires a contractile force on the membrane, and they differ primarily in the orientation of the contractile apparatus. If phagocytosis evolved first, it is easy to imagine this system being co-opted later for cytokinesis, at first assisting FtsZ and eventually replacing it entirely. However there is also reason to think that cytokinesis may have been the first to utilize the actin-myosin apparatus, with phagocytosis evolving later. This is addressed in the next section.

\section{Some comments on cell size}

Bacteria are generally limited to a diameter of $\sim 1 \mu \mathrm{m}$. A major limitation on their size may be diffusion of metabolites from outside the cell to its surface. "A diffusion-limited bacterium could potentially increase its specific rate of metabolism fourfold if the cell diameter were only half as large."(76) There are some remarkable exceptions, and bacterial species with diameters of 100-200 $\mu \mathrm{m}$ and lengths of several hundred $\mu \mathrm{m}$ are known.(76) The largest ones are symbionts in the gut of certain animals, and they may solve the diffusion problem by inhabiting nutrient-rich environments.

At one point, my colleagues and I were intrigued that these giant bacteria might provide opportunities to study giant $Z$ rings. However, in at least two cases that have been studied, the bacteria cheat and deny us this opportunity. Metabacterium polyspora and Epulopiscium $s p p$. do not divide by binary fission. Instead they form endospores, and they do this at the cell poles where the $Z$ ring need be no more than $\sim 1 \mu \mathrm{m}$ in diameter to pinch off the tiny endospores. $(77,78)$

There is no known bacterial $\mathrm{Z}$ ring larger than about $1 \mu \mathrm{m}$ diameter. Chloroplast $\mathrm{Z}$ rings can be much larger, but these organelles have additional mechanisms assisting division. This raises the possibility that the $\mathrm{Z}$ ring may not be able to function for division beyond a $1 \mu \mathrm{m}$ diameter. If so, this would be a serious limitation on the size of any cell using a $\mathrm{Z}$ ring for cytokinesis. The actin-myosin-based eukaryotic cytokinesis machine can function at much larger diameters. Thus replacing the $\mathrm{Z}$ ring with actin-based cytokinesis may have been a key step in permitting eukaryotic cells to achieve their much larger size. Phagocytosis itself may have evolved after cytokinesis by a rotation of the cytokinetic actin-myosin ring. It is hard to imagine the developing eukaryote as a phagocytic predator until it had acquired a size larger than its prey.

\section{Conclusion}

The proteins FtsZ and MreB have been described as the "prokaryotic origins of the cytoskeleton". The term prokaryote is generally understood to comprise bacteria and archaea. I argue here that these cytoskeletal proteins probably evolved even earlier, in the common ancestor of bacteria, archaea and eukarya. FtsZ in particular is an ancient protein. FtsZ and MreB originally functioned for cytokinesis and cell shape, respectively, and they have maintained these functions across bacteria and archaea. The 40-50\% sequence identity across different species represents the limit of divergence that is consistent with maintaining these functions. In eukarya, however, FtsZ and MreB lost their original functions and acquired new 
ones. Optimizing these new functions would require substantial co-evolution with motor molecules and binding proteins. Once tubulin and actin had optimized their assembly mechanisms and their interactions with multiple motors and binding proteins, their sequences were largely frozen as they spread in evolving eukaryotic species.

\section{References}

1. Bermudes D, Hinkle G, Margulis L. Do prokaryotes contain microtubules? Microbiol Rev 1994;58:387-400. [PubMed: 7968920]

2. RayChaudhuri D, Park JT. Escherichia coli cell-division gene ftsZ encodes a novel GTP-binding protein. Nature 1992;359:251-254. [PubMed: 1528267]

3. de Boer P, Crossley R, Rothfield L. The essential bacterial cell division protein FtsZ is a GTPase. Nature 1992;359:254-256. [PubMed: 1528268]

4. Mukherjee A, Dai K, Lutkenhaus J. Escherichia coli cell division protein FtsZ is a guanine nucleotide binding protein. Proc Natl Acad Sci USA 1993;90:1053-1057. [PubMed: 8430073]

5. Erickson HP, Taylor DW, Taylor KA, Bramhill D. Bacterial cell division protein FtsZ assembles into protofilament sheets and minirings, structural homologs of tubulin polymers. Proc Natl Acad Sci USA 1996;93:519-523. [PubMed: 8552673]

6. Löwe J, Amos LA. Crystal structure of the bacterial cell-division protein FtsZ. Nature 1998;391:203206. [PubMed: 9428770]

7. Nogales E, Wolf SG, Downing KH. Structure of the alpha beta tubulin dimer by electron crystallography. Nature 1998;391:199-203. [PubMed: 9428769]

8. Bork P, Sander C, Valencia A. An ATPase domain common to prokaryotic cell cycle proteins, sugar kinases, actin, and hsp70 heat shock proteins. Proc Natl Acad Sci USA 1992;89:7290-7294. [PubMed: 1323828]

9. Kabsch W, Mannherz HG, Suck D, Pai EF, Holmes KC. Atomic structure of the actin: DNase I complex. Nature 1990;347:37-44. [PubMed: 2395459]

10. Doolittle RF, York AL. Bacterial actins? An evolutionary perspective. Bioessays 2002;24:293-296. [PubMed: 11948613]

11. van den Ent F, Lowe J. Crystal structure of the cell division protein FtsA from Thermotoga maritima. Embo J 2000;19:5300-5307. [PubMed: 11032797]

12. Carettoni D, Gomez-Puertas P, Yim L, Mingorance J, Massidda O, et al. Phage-display and correlated mutations identify an essential region of subdomain $1 \mathrm{C}$ involved in homodimerization of Escherichia coli FtsA. Proteins 2003;50:192-206. [PubMed: 12486713]

13. Pichoff $\mathrm{S}$, Lutkenhaus J. Tethering the $\mathrm{Z}$ ring to the membrane through a conserved membrane targeting sequence in FtsA. Mol Microbiol 2005;55:1722-1734. [PubMed: 15752196]

14. Lara B, Rico AI, Petruzzelli S, Santona A, Dumas J, et al. Cell division in cocci: localization and properties of the Streptococcus pneumoniae FtsA protein. Mol Microbiol 2005;55:699-711. [PubMed: 15660997]

15. Jones LJ, Carballido-Lopez R, Errington J. Control of cell shape in bacteria: helical, actin-like filaments in Bacillus subtilis. Cell 2001;104:913-922. [PubMed: 11290328]

16. van den Ent F, Amos LA, Lowe J. Prokaryotic origin of the actin cytoskeleton. Nature 2001;413:39_ 44. [PubMed: 11544518]

17. Ausmees N, Kuhn JR, Jacobs-Wagner C. The bacterial cytoskeleton: an intermediate filament-like function in cell shape. Cell 2003;115:705-713. [PubMed: 14675535]

18. Walsh DA, Doolittle WF. The real 'domains' of life. Curr Biol 2005;15:R237-240. [PubMed: 15823519]

19. Doolittle WF. Revolutionary concepts in evolutionary cell biology. Trends Biochem Sci 1980;5:146149.

20. Hartman H, Fedorov A. The origin of the eukaryotic cell: a genomic investigation. Proc Natl Acad Sci USA 2002;99:1420-1425. [PubMed: 11805300]

21. Kurland CG, Collins LJ, Penny D. Genomics and the irreducible nature of eukaryote cells. Science 2006;312:1011-1014. [PubMed: 16709776] 
22. Martin W, Muller M. The hydrogen hypothesis for the first eukaryote. Nature 1998;392:37-41. [PubMed: 9510246]

23. Embley TM, Martin W. Eukaryotic evolution, changes and challenges. Nature 2006;440:623-630. [PubMed: 16572163]

24. Cavalier-Smith T. The phagotrophic origin of eukaryotes and phylogenetic classification of Protozoa. Int J Syst Evol Microbiol 2002;52:297-354. [PubMed: 11931142]

25. Stanier RY. Some aspects of the biology of cells and their possible evolutionary significance. Symp Soc Gen Microbiol 1970;20:1-38.

26. de Duve C. The birth of complex cells. Sci Am 1996;274:50-57. [PubMed: 8907651]

27. Doolittle WF. Uprooting the tree of life. Sci Am 2000;282:90-95. [PubMed: 10710791]

28. Doolittle RF. The origins and evolution of eukaryotic proteins. Phil Trans R Soc Lond B 1995;349:235-240. [PubMed: 8577833]

29. Faguy DM, Doolittle WF. Cytoskeletal proteins: the evolution of cell division. Curr Biol 1998;8:R338-341. [PubMed: 9601632]

30. Vaughan S, Wickstead B, Gull K, Addinall SG. Molecular evolution of FtsZ protein sequences encoded within the genomes of archaea, bacteria, and eukaryota. J Mol Evol 2004;58:19-29. [PubMed: 14743312]

31. Keeling PJ, Doolittle WF. Alpha-tubulin from early-diverging eukaryotic lineages and the evolution of the tubulin family. Mol Biol Evol 1996;13:1297-1305. [PubMed: 8952074]

32. Katiyar SK, Edlind TD. Entamoeba histolytica encodes a highly divergent b-tubulin. J Euk Microbiol 1996;43:31-34. [PubMed: 8563707]

33. Davis BK. Molecular evolution before the origin of species. Prog Biophys Mol Biol 2002;79:77-133. [PubMed: 12225777]

34. Nogales E, Downing KH, Amos LA, Lowe J. Tubulin and FtsZ form a distinct family of GTPases. Nature Structural Biology 1998;5:451-458.

35. Inclan YF, Nogales E. Structural models for the self-assembly and microtubule interactions of gamma, delta, and epsilon-tubulin. J Cell Sci 2001;114:413-422. [PubMed: 11148142]

36. Lowe J, Li H, Downing KH, Nogales E. Refined Structure of alphabeta-Tubulin at 3.5 A Resolution. J Mol Biol 2001;313:1045-1057. [PubMed: 11700061]

37. Mukherjee A, Lutkenhaus J. Guanine nucleotide-dependent assembly of FtsZ into filaments. Journal of Bacteriology 1994;176:2754-2758. [PubMed: 8169229]

38. Erickson HP, Stoffler D. Protofilaments and rings, two conformations of the tubulin family conserved from bacterial FtsZ to alpha/beta and gamma tubulin. J Cell Biol 1996;135:5-8. [PubMed: 8858158]

39. Löwe J, Amos LA. Tubulin-like protofilaments in Ca2+-induced FtsZ sheets. EMBO J 1999; 18:23642371. [PubMed: 10228151]

40. Oliva MA, Cordell SC, Lowe J. Structural insights into FtsZ protofilament formation. Nat Struct Mol Biol 2004;11:1243-1250. [PubMed: 15558053]

41. Nogales E, Whittaker M, Milligan RA, Downing KH. High-resolution model of the microtubule. Cell 1999;96:79-88. [PubMed: 9989499]

42. Erickson HP. Atomic structures of tubulin and FtsZ. Trends in Cell Biol 1998;8:133-137. [PubMed: 9695825]

43. Dai K, Mukherjee A, Xu Y, Lutkenhaus J. Mutations in ftsZ that confer resistance to SulA affect the interaction of FtsZ with GTP. J Bacteriol 1994;176:130-136. [PubMed: 8282688]

44. Redick SD, Stricker J, Briscoe G, Erickson HP. Mutants of FtsZ Targeting the Protofilament Interface: Effects on Cell Division and GTPase Activity. J Bacteriol 2005;187:2727-2736. [PubMed: 15805519]

45. Oegema K, Wiese C, Martin OC, Milligan RA, Iwamatsu A, et al. Characterization of Two Related Drosophila gamma-tubulin Complexes that Differ in Their Ability to Nucleate Microtubules. J Cell Biol 1999;144:721-733. [PubMed: 10037793]

46. Stricker J, Erickson HP. In vivo characterization of Escherichia coli ftsZ mutants: effects on Z-ring structure and function. J Bacteriol 2003;185:4796-4805. [PubMed: 12896999]

47. Reijo RA, Cooper EM, Beagle GJ, Huffaker TC. Systematic mutational analysis of the yeast gamma-, delta- and epsilon-tubulin gene. Molec Biol Cell 1994;5:29-43. [PubMed: 8186463] 
48. Richards KL, Anders KR, Nogales E, Schwartz K, Downing KH, et al. Structure-function relationships in yeast tubulins. Mol Biol Cell 2000;11:1887-1903. [PubMed: 10793159]

49. Lichtarge O, Bourne HR, Cohen FE. An evolutionary trace method defines binding surfaces common to protein families. J Mol Biol 1996;257:342-358. [PubMed: 8609628]

50. Armon A, Graur D, Ben-Tal N. ConSurf: an algorithmic tool for the identification of functional regions in proteins by surface mapping of phylogenetic information. J Mol Biol 2001;307:447-463. [PubMed: 11243830]

51. Valdar WS, Thornton JM. Protein-protein interfaces: analysis of amino acid conservation in homodimers. Proteins 2001;42:108-124. [PubMed: 11093265]

52. Osawa M, Erickson HP. FtsZ from divergent foreign bacteria can function for cell division in Escherichia coli. J Bacteriol 2006;188:7132-7140. [PubMed: 17015652]

53. Kull FJ, Vale RD, Fletterick RJ. The case for a common ancestor: kinesin and myosin motor proteins and G proteins. J Muscle Res Cell Motil 1998;19:877-886. [PubMed: 10047987]

54. Richards TA, Cavalier-Smith T. Myosin domain evolution and the primary divergence of eukaryotes. Nature 2005;436:1113-1118. [PubMed: 16121172]

55. Jenkins C, Samudrala R, Anderson I, Hedlund BP, Petroni G, et al. Genes for the cytoskeletal protein tubulin in the bacterial genus Prosthecobacter. Proc Natl Acad Sci USA 2002;99:17049-17054. [PubMed: 12486237]

56. Sontag CA, Staley JT, Erickson HP. In vitro assembly and GTP hydrolysis by bacterial tubulins BtubA and BtubB. J Cell Biol 2005;169:233-238. [PubMed: 15851515]

57. Schlieper D, Oliva MA, Andreu JM, Lowe J. Structure of bacterial tubulin BtubA/B: Evidence for horizontal gene transfer. Proc Natl Acad Sci USA 2005;102:9170-9175. [PubMed: 15967998]

58. McKean PG, Vaughan S, Gull K. The extended tubulin superfamily. J Cell Sci 2001;114:2723-2733. [PubMed: 11683407]

59. Tinsley E, Khan SA. A novel FtsZ-like protein is involved in replication of the anthrax toxin-encoding pXO1 plasmid in Bacillus anthracis. J Bacteriol 2006;188:2829-2835. [PubMed: 16585744]

60. Tang M, Bideshi DK, Park HW, Federici BA. Minireplicon from pBtoxis of Bacillus thuringiensis subsp. israelensis. Appl Environ Microbiol 2006;72:6948-6954. [PubMed: 16936050]

61. Roeben A, Kofler C, Nagy I, Nickell S, Hartl FU, et al. Crystal structure of an archaeal actin homolog. J Mol Biol 2006;358:145-156. [PubMed: 16500678]

62. Hara F, Yamashiro K, Nemoto N, Ohta Y, Yokobori SI, et al. An actin homolog of the archaeon Thermoplasma acidophilum that retains the ancient characteristics of eukaryotic actin. J Bacteriol 2006;189:2039-2045. [PubMed: 17189356]

63. Hixon WG, Searcy DG. Cytoskeleton in the archaebacterium Thermoplasma acidophilum? Viscosity increase in soluble extracts. Biosystems 1993;29:151-160. [PubMed: 8374067]

64. Moller-Jensen J, Jensen RB, Lowe J, Gerdes K. Prokaryotic DNA segregation by an actin-like filament. Embo J 2002;21:3119-3127. [PubMed: 12065424]

65. Garner EC, Campbell CS, Weibel DB, Mullins RD. Reconstitution of DNA segregation driven by assembly of a prokaryotic actin homolog. Science 2007;315:1270-1274. [PubMed: 17332412]

66. Becker E, Herrera NC, Gunderson FQ, Derman AI, Dance AL, et al. DNA segregation by the bacterial actin AlfA during Bacillus subtilis growth and development. Embo J 2006;25:5919-5931. [PubMed: 17139259]

67. Gitai Z, Dye NA, Reisenauer A, Wachi M, Shapiro L. MreB actin-mediated segregation of a specific region of a bacterial chromosome. Cell 2005;120:329-341. [PubMed: 15707892]

68. Kruse T, Blagoev B, Lobner-Olesen A, Wachi M, Sasaki K, et al. Actin homolog MreB and RNA polymerase interact and are both required for chromosome segregation in Escherichia coli. Genes Dev 2006;20:113-124. [PubMed: 16391237]

69. Dominguez R. Actin-binding proteins-a unifying hypothesis. Trends Biochem Sci 2004;29:572578. [PubMed: 15501675]

70. Pollard TD, Blanchoin L, Mullins RD. Molecular mechanisms controlling actin filament dynamics in nonmuscle cells. Annu Rev Biophys Biomol Struct 2000;29:545-576. [PubMed: 10940259] 
71. Galkin VE, VanLoock MS, Orlova A, Egelman EH. A new internal mode in F-actin helps explain the remarkable evolutionary conservation of actin's sequence and structure. Curr Biol 2002;12:570575. [PubMed: 11937026]

72. Mullins, RD. Actin-Related Proteins.. In: Lane, WJ.; La, MD., editors. Encyclopedia of Biological Chemistry. Elsevier; New York: 2004. p. 27-33.

73. Maciver, S. 2007. Actin Related Proteins (ARPs) Web Page. http://www.bms.ed.ac.uk/research/others/smaciver/encyclop/ABPA/Actin-Related\%20Proteins.htm 2007

74. Cavalier-Smith T. The neomuran origin of archaebacteria, the negibacterial root of the universal tree and bacterial megaclassification. Int J Syst Evol Microbiol 2002;52:7-76. [PubMed: 11837318]

75. Uenoyama A, Miyata M. Gliding ghosts of Mycoplasma mobile. Proc Natl Acad Sci USA 2005;102:12754-12758. [PubMed: 16126895]

76. Schulz HN, Jorgensen BB. Big bacteria. Annu Rev Microbiol 2001;55:105-137. [PubMed: 11544351]

77. Angert ER, Losick RM. Propagation by sporulation in the guinea pig symbiont Metabacterium polyspora. Proc Natl Acad Sci USA 1998;95:10218-10223. [PubMed: 9707627]

78. Robinow C, Angert ER. Nucleoids and coated vesicles of "Epulopiscium" spp. Arch Microbiol 1998;170:227-235. [PubMed: 9732436]

79. Cordell SC, Robinson EJ, Lowe J. Crystal structure of the SOS cell division inhibitor SulA and in complex with FtsZ. Proc Natl Acad Sci USA 2003;100:7889-7894. [PubMed: 12808143] 


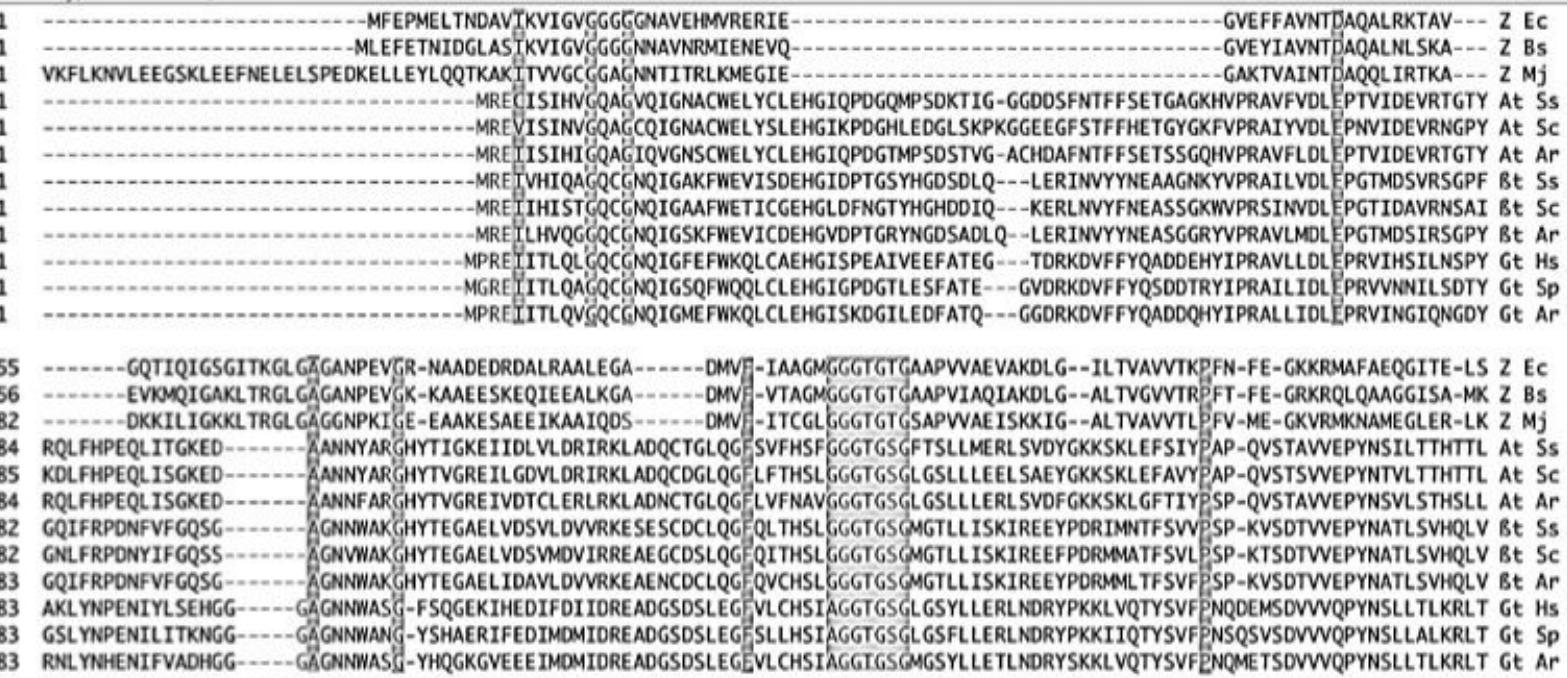

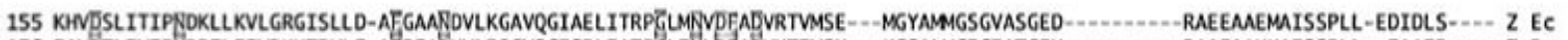

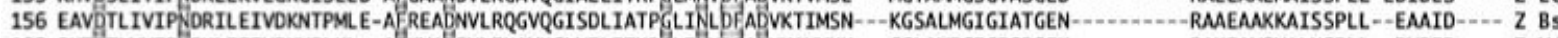

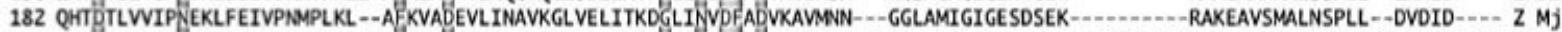
196 EHSDCAFMVD EATYDICRRNLDIERPTYTNL NRLIGQIVSSITASLRFDGALNVDITE FQTNLVPYPRAHFPLATYAPVISAE---KAYHEQLSVAEITNACFEPANQMVKCDPRHG-- At SS 197 EHADCTFMVD EAIYDMCKRNLDIPRPS EANL NNLIAQVVSSVTASLRFDGSLNVDLNEFQTNLVPYPRIHFPLVSYSPVLSKS---KAFHESNSVSEITNACFEPGNQMVKCDPRDG-- AT SC 196 EHTRVVLLONEATYDICRRSLDIERPT SNL RRLISQTISSLTTSLRFDGAINVDITEFQTNLVPYPRIHFMLSSYAPVISSA---KAYHEQFSVPEITTSVFEPSNMMAKCDPRHG-- AT Ar 194 ENTPETYCID EALYDICFRTLKLTTPT GDLNHLVSATMSGVTTCLRFPGQLNADLRKLAVIMNPFPRLHFFMPGFAPLTSRG---SQQYRALTVPELTQQMFDAKNMUACDPRHG-- Bt SS 194 EHSDETFCIDNEALYDICQRTLKLNQPSY GDLNNLVSSVMSGVTTSLRYPGQLNSDLRKLAVNLVPFPRLHFFMVGYAPLTAIG---SQSFRSLTVPELTQQMFDAKNMMAAADPRG-- Bt SC 195 ENADEEONLD EALYDICFRTLKLSTPS GDL HLISATMSGVTCSLRFPGQLNSDLRKLAVNLIPFPRLHFFMVGFAPLTSRG---SQQYISLTVPELTQQMWDAKMMICAADPRHG-- BE AR 197 QNAD̈CLVVL D TALNRIATDRLHIQNPS SQIN QLVSTIMSASTTILRYPGYMNNDLIGLIASLIPTPRLHFLMTGYTPLTTDQ--SVASVRKTTVLDVMRRLLQPKNVMVSTGRDRQTN GE HS 197 LNADSSWVLDNALLAHIAADRLHTQNPT HQQNQLVSTVMSASTTTLRYPGYMNNDL VSSIIASLIPSPRCHFLLTSYTPFTNQQVEEAKAIRKTTVLDVMRLLLLPKNQMVSVNPSKKS- GE SP 197 LNABCWVLDNTALGRIAVERLHLTNPTEAQTISLVSTVMSASTTTLRYPGYMNNDLVGLLASLIPTPRCHFLMTGYTPLTVER--QANVIRKTTVLDVMRRLLQTKNIMVSSYARNKE- Gt AP

256 -GARGVLVWIT-AGFDLRLDEFETVGNTIRAFAS----DNATVIGTSLDPDM------NDELRVTVVATGIGMOKRPEITLVTNKQVQQPVMDRYQQHGMAPLTQEQKPVAKVVN Z EC

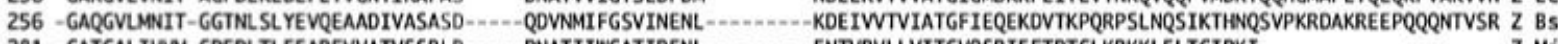
281 -GATGALIHMM-GPEDLTLEEAREVVATVSSRLD-...-PNATIIWGATIDENL-.......-ENTVRVLLVITGVQSRIEFTDTGLKRKKLELTGIPKI Z

311 -KYMACCLLYR---GOVVPKDVNMAIATIKTKRTIOFVDWCPTGFKVGINYEPPTVVPGGDLAKVQRAVOMLSNTTAIAEAWARLDHKFDLMYAKRAFVHWYVGEGMEEGEFSEAREDMA AT SS 312 -KYMATCLLYR---GDVVTRDVQRAVEQVKNKKTVQLVDWCPTGFKIGICYEPPTATPNSQLATVDRAVCMLSNTTS IAEAWKRIDRKFDLMYAKRAFVHWYVGECMEEGEFTEAREDLA AT SC 311 -KYMACCLMYR---GDVVPDVWTAVAAIKAKRTIQFVDWCPTGFKCGINYQPPSWVPGDLAKVQRAVOMISNNTAVAEVFSRIDHKFDLMYSKRAFVHWYVGEGEEGEFSEAREDLA AT AR 309 -RYLTVAAVFR--GRUSMKEVDEQMLNVQNKNSSYFVEWIPNNVKTAVCDIPP_.....-RGLKMSATF IGNSTAIQELFKRISEQFTAMFRRKAFLHWYTGECMDEMEFTEAESNMN BE SS 309 -RYLTVAAFFR---GKVSVKEVEDEMHKVQSKNSDYFVEWIPNNVQTAVCSVAP .......-QGLDMATFF IANSTSIQELFKRVGDQFSAMFKRKAFLHWYTSECMDELEFSEAESNMN BE SC 310 -RYLTASAMFR -.-GKUSTKEVDEQILNVQNKNSSYFVEWIPNNVKSSVCDIPP........-TGIKMASTFVGNSTS IQEMFRRVSEQFTAMFRRKAFLHWYTGECMDEMEFTEAESNMN BE AR 315 HCYIAILNIIQ---GEVDPTQWHKSLQRIRERKLANFIPWGPASIQVAL SRKSPYLP-----SAHRVSGLMMANHTSISSLFERTCRQYDKLRKREAFLEQFRKEDMFKDNFDEMDTSRE GE HS 316 -CFISILDIRQ--GEADPADVHKSLLRIRERRYASFIPWGPASIQVALSKKSPYIK----TNHRVSGLMLANHTSIASLFKRTLDQYDRLRKRNAFLEQYKKEAIFE-DDLNEFDSSR Gt SP 314 ASQAKYISILNIIQGEVDPTQVHESLQRIRERKLVNFIEWGPASIQVAL SKKSPYVQ-----TAHRVSGLMLASHTSIRHLFSKCLSQYOKLRKKQAFLDNYRKFPMFADNDLSEFDESR GE AP

360 DNAPQTAKEPOYLDIPAFLRKQAD

360 HT-SQPA--DDTLDIPTFLRNRNKRG

364

427 ALEKDYEEVGVDSVE-GEGEEEGEEY

428 ALERDYIEVGADSYA-EEEEF

427 ALEKDYEEVGGEGAE-DDOEEGDEY

417 DLVSEYQQYQDATADEQGEFEEEGEEDEA

417 DLVSEYQQYQEATVEDDEEVDENGDFGAPQNQDEPITENFE

418 DLVSEYQQYQDATADEEDEYDEEEEQVYES

427 IVQQL-IDEYHAATRPOYISWGTQEQ

426 DWADLINEYEACEDPNYLSL

429 DIIESLVDEYKACESPDYIKWGMEDPEQLMTGEGNASGVVDPKLAF

$Z \mathrm{EC}$

$\mathrm{Z}$ Bs

$\mathrm{Z} M \mathrm{Mj}$

At $\mathrm{Ss}$

At $\mathrm{Sc}$

At Ar

Bt Ss

Bt Sc

Bt Ar

Gt Hs

Gt Sp

Gt Ar

Figure 1.

Structure-based sequence alignment of FtsZ, $\alpha, \beta$ and $\gamma$ tubulins. Amino acids judged to be highly conserved are indicated by grey shading, and are detailed in Table 1 and Fig. 2. The FtsZ sequences beyond G312 of the E. coli sequence are highly divergent even among FtsZs and show no relationship to tubulins. 


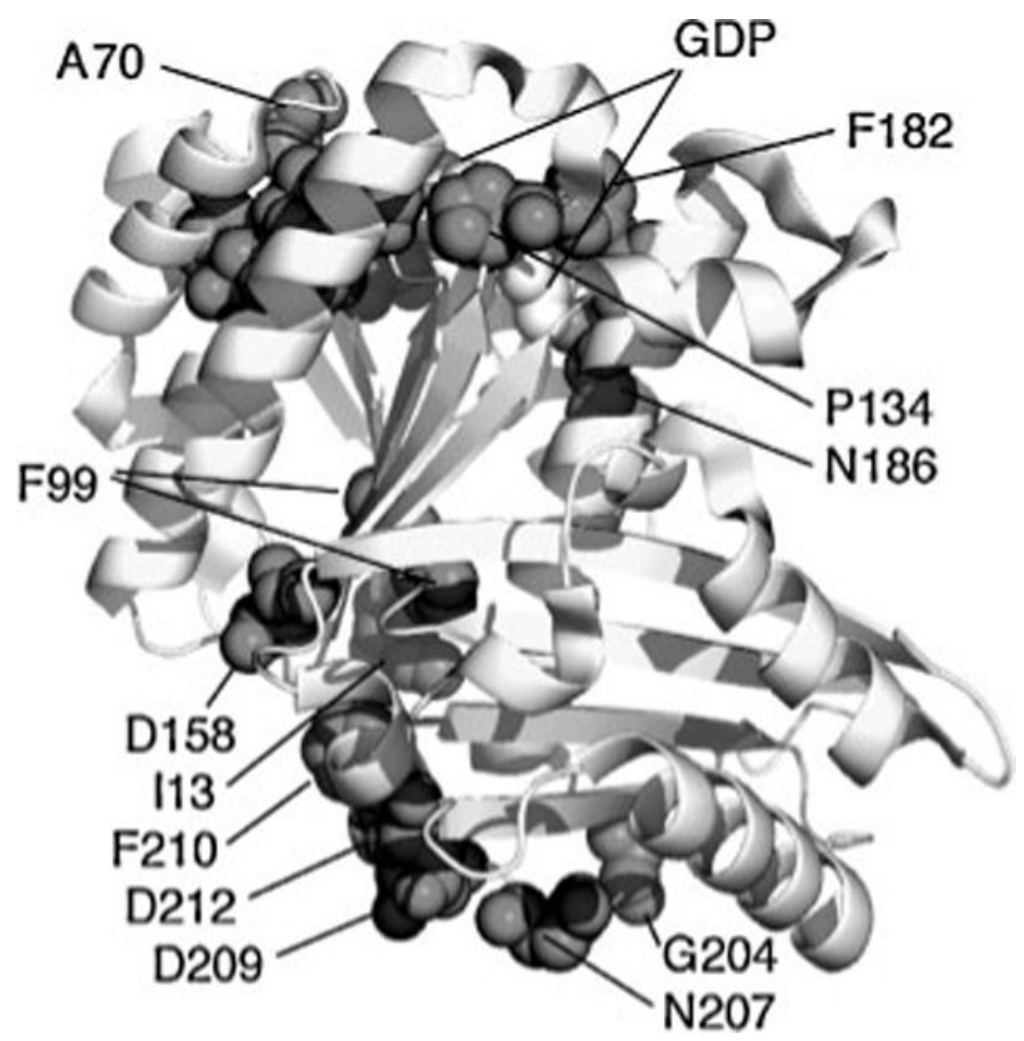

Figure 2.

The amino acids judged as highly conserved in Fig. 1 and Table 1 are shown in dark spacefilling on the structure of FtsZ from Pseudomonas aeruginosa(79) (PDB file 1OFU). GDP is white spacefill (mostly hidden). Several amino acids forming the GDP-binding pocket are indicated, but most are too close for separate identification. The figure was prepared with the program PyMol (DeLano Scientific LLC). 
Table 1

Amino acids conserved in FtsZ and alpha, beta and gamma tubulins

\begin{tabular}{|c|c|c|}
\hline E. coli FtsZ aa & pig $\alpha$ tub aa & Location and comments \\
\hline $\mathrm{I} 13$ & $\mathrm{C} 4$ & Core, buried \\
\hline G19 & G10 & Top; GTP \\
\hline G22 & G13 & Top \\
\hline D45 & E71 & Top; GTP (E in tubulins) \\
\hline A70 & A99 & Top; GTP \\
\hline G77 & G106 & Top \\
\hline F99 & $\mathrm{F} 135$ & Core, completely buried \\
\hline G105 & G142 & Top; GTP; A in $\gamma$ tub \\
\hline G106 & G143 & Top; GTP \\
\hline G107 & G144 & Top; GTP \\
\hline T108 & $\mathrm{T} 145$ & Top; GTP \\
\hline G109 & G146 & Top; GTP \\
\hline $\mathrm{T} 110$ & $\mathrm{~S} 147$ & Top; $\mathrm{S}$ in all tubulins \\
\hline G111 & G148 & Top \\
\hline $\mathrm{P} 134$ & P173 & Top; GTP \\
\hline D158 & D199 & Left side \\
\hline N165 & N206 & Top; GTP \\
\hline F182 & Y224 & Top; GTP; Y/F in tubulins \\
\hline N186 & $\mathrm{N} 228$ & Top; GTP; D in half of FtsZs \\
\hline G204 & G246 & Bottom; synergy/T7 \\
\hline N207 & $\mathrm{N} 249$ & Bottom; GTP-synergy/T7 \\
\hline D209 & D251 & Bottom; GTP-synergy/T7 \\
\hline $\mathrm{F} 210$ & $\mathrm{~L} 252$ & Bottom; synergy/T7; L in all tubulins \\
\hline D212 & $\mathrm{E} 254$ & Bottom; GTP-synergy/T7; E in $\alpha, \mathrm{K}$ in $\beta$, and G/S in $\gamma$ tubulin \\
\hline
\end{tabular}

These amino acids are highlighted in the sequence alignment of Fig. 1, and shown on the 3-D structure in Fig. 2. GTP indicates that this amino acid was identified as contacting the GTP (Fig. 3 in Lowe et al.(36); see Oliva et al.(40) for FtsZ A70). Each of these amino acids is highly conserved in FtsZ. Some tubulins show conservative or function disruption substitutions as noted in column 3. 